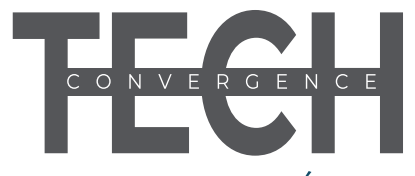

REVISTRCIENTÍFICA

Marzo-Agosto 2020

Vol. 4, No.1,24-29

ISSN 2737-6087

\title{
PROPUESTA DE ESTANDARIZACIÓN EN LA SECCIÓN “TRUCHAS Y MARISCOS" DE LA HOSTERÍA DOS CHORRERAS
}

\section{Richard Antonio Martínez Villegas ${ }^{1}$, Daniel Rolando Fernández Sánchez ${ }^{2}$, Evelyn Cristina Narváez Solís ${ }^{3}$}

\author{
${ }^{1}$ Instituto de Tecnologías Particular Sudamericano \\ rimartinez@sudamericano.edu.ec (iD https://orcid.org/0000-0001-6655-9972 \\ ${ }^{2}$ Instituto de Tecnologías Particular Sudamericano \\ drfernandez@sudamericano.edu.ec \\ ${ }^{1}$ Instituto de Tecnologías Particular Sudamericano \\ ecnarvaez@sudamericano.edu.ec
}

\section{RESUMEN}

Esta investigación nace de la necesidad de mejorar y optimizar la producción de alimentos y bebidas de la sección "Trucha y Mariscos" del menú de la hostería Dos Chorreras; para ello, se ha utilizado una metodología con enfoque mixto, que hace uso de diferentes técnicas de campo, específicamente la entrevista personal, la encuesta al personal operativo y, sobre todo, la observación descriptiva; a través de ellas, se determinó alteraciones en el proceso de producción, demostrando la variación de características organolépticas de las elaboraciones y la variación en cuanto al gramaje de los alimentos al momento de realizar los montajes de los platos. A partir de estos resultados, se ha delineado como objetivo generar una propuesta de estandarización de los platillos. La socialización al personal operativo de dicha propuesta permitió mejorar la producción y facilitó una guía para la elaboración de alimentos. Esto evitará tanto el desperdicio de materia prima como los cambios de características organolépticas; ayudará, asimismo, a realizar un montaje homogéneo de los platos.

Palabras Clave: cocina de autor, estandarización, plato fuerte, producción, propuestas gastronómicas, trucha
Fecha de recepción: 15 de noviembre de 2019 Fecha de aceptación: 8 de enero de 2020

\section{ABSTRACT}

This research was born from the need to improve and optimize the production of food and beverages in the "Trout and Seafood" section of the "Dos Chorreras" inn menu; For it, a methodology with a mixed approach has been used, which makes use of different field techniques, specifically the personal interview, the survey of operational personnel and, above all, descriptive observation; Through them, alterations in the production process were determined, demonstrating the variation of organoleptic characteristics of the preparations and the variation in terms of the weight of the food at the time of assembling the dishes. From these results, the objective has been outlined to generate a proposal for the standardization of the dishes. The socialization to the operational personnel of the said proposal allowed to improve production and provided a guide for food preparation. This will avoid both the waste of raw material as the changes of organoleptic characteristics; It will also help to make a homogeneous assembly of the plates. Keywords: signature cuisine, standardization, main dish, production, gastronomic proposals, trout

Forma sugerida de citar: Martínez Villegas, R. A., Fernández Sánchez, D. R., Narváez Solís, E. C. (2020). Propuesta de estandarización en la sección "Truchas y Mariscos" de la hostería Dos Chorreras. Convergence Tech Revista Científica. 4(1), 24-29. 


\section{INTRODUCCIÓN}

$\mathbf{U}$

$\mathrm{n}$ proceso de producción gastronómico sin estandarización representa una seria carencia para la empresa, ya que genera deficiencias en las características organolépticas del plato. A partir de la observación de campo, se determina que éste es precisamente el problema del área de cocina de la hostería Dos Chorreras, ya que no cuenta con un menú debidamente estandarizado; para solventarlo, la presente investigación propone una herramienta de apoyo al personal operativo del área de cocina, a través de una breve guía de producción para los administradores de la hostería. Para ello, se inicia por plantear la pregunta "icómo mejoraría la producción del menú con la aplicación de una estandarización de procesos dentro del restaurante?".

Para responder a esta pregunta, este proyecto empieza por mejorar eficazmente la elaboración de los platillos ofertados, estandarizando las recetas, ya que se considera de antemano que su implementación resultará en una producción factible, en una mayor preservación de las características organolépticas y en un montaje homogéneo; posibilitará, a su vez, la optimización de tiempos, costos y materia prima involucrados en los procesos de manufactura del restaurante

Ahora bien, si se considera que la estandarización es el proceso de ajustar o adaptar características de un producto, servicio o procedimiento, con el objetivo de que éstas se asemejen a un tipo, modelo o norma en común, permitiendo la creación de estándares que establecen las características comunes con las que deben cumplir los productos y que son respetadas en diferentes partes del mundo (Secretaria de Economía, 2015), se comprenderá que una correcta producción de alimentos esté determinada por el cumplimiento de una ficha estándar que permita tanto la descripción de cada paso a seguir, como las cantidades, el peso, la mise en place, las temperaturas, las técnicas y el tiempo necesarios. Como señala Rodríguez (2018, párr. 2), "la Ficha Técnica (o Ficha de Receta) es la herramienta fundamental para que tu equipo de cocina siga al pie de la letra tus recetas y ofrecer así a tus clientes la misma experiencia gastronómica siempre".

\section{DESARROLLO}

\section{Organización de la cocina de la hostería Dos Chorreras}

l plantearse este trabajo como una propuesta
de estandarización que mejore el servicio de
cocina de la hostería Dos Chorreras, es importante revisar brevemente la organización de su cocina. Así pues, lo primero que se evidencia es que tiene la infraestructura adecuada y el personal capacitado para receptar fichas estandarizadas y laborar en todas las áreas de la cocina; en cada una de estas áreas, por lo demás, laboran dos personas.

Las áreas correspondientes a la producción se dividen en las secciones: entradas, fuertes y pastelería, cada

Figura $1 »$ Organigrama de Cocina.

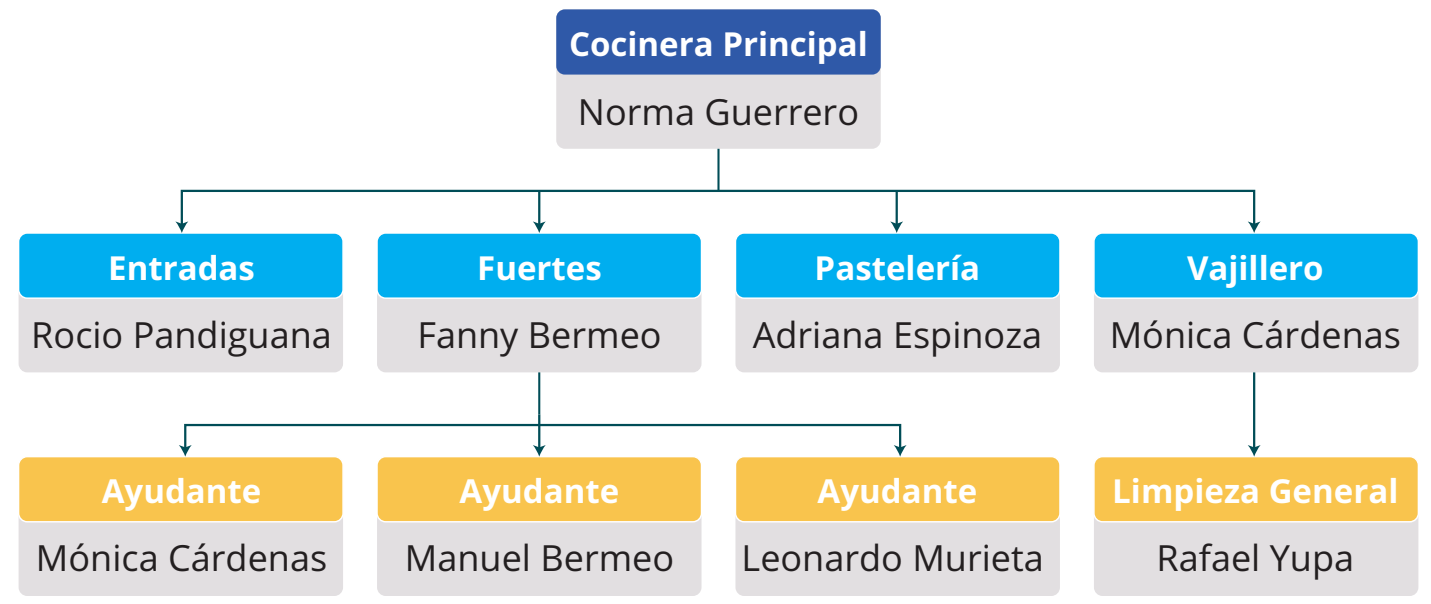

Fuente: Fernández y Narváez (2018). 
una de las cuales están correctamente equipadas con maquinarias y segmentos de producción suficientes para los diferentes espacios culinarios.

De hecho, tal y como se demuestra en el grafico 1, el personal operativo del área de cocina trabaja con órdenes directas de la cocinera principal, encargada de capacitar e instruir al personal para las diferentes áreas.

El restaurante, al ser un espacio con un alto índice de demanda, cuanta con una bodega de almacenamiento de alimentos no perecibles y perecibles, que despacha semanalmente los alimentos requeridos por el área de cocina.

\section{Metodología}

Para la obtención y análisis de información se utilizó la metodología mixta ya que permite analizar la información desde un punto de vista cuantitativo como cualitativo. Al respecto Chen, citado por Hernández (2010, párr. 9), dice:

Las investigaciones con enfoque mixto consisten en la integración sistemática de los métodos cuantitativo y cualitativo en un solo estudio con el fin de obtener una "fotografía" más completa del fenómeno. Pueden ser conjuntados de tal manera que las aproximaciones cuantitativa y cualitativa conserven sus estructuras y procedimientos originales ("forma pura de los métodos mixtos"). Alternativamente, estos métodos pueden ser adaptados, alterados o sintetizados para efectuar la investigación y lidiar con los costos del estudio ("forma modificada de los métodos mixtos").

Así pues, para esta investigación se utilizaron técnicas tales como: técnica de campo, observación, entrevista y encuesta. Además, se empleó la técnica de laboratorio permitiendo el desarrollo de los seis platos fuertes de autor; en este sentido, vale decir que, para el desarrollo de esta última técnica, se contó con la disposición de los equipos y utensilios necesarios para la realización de los platos y, a su vez, se obtuvo información precisa y real para el correcto llenado de la base de datos, fichas estándar y bitácoras a realizarse -estas últimas, conformadas por la base de datos, ficha estándar, bitácoras-. Estas técnicas sirven como respaldo de las pruebas gastronómicas que se han realizado para la aprobación de los seis platos de autor.

\section{Análisis e interpretación de datos}

Tal como mencionan Baculima y Quizhpi (2017, p. 27), en el Ecuador la trucha ha sido frecuentemente menospreciada por las restringidas elaboraciones que se pueden realizar de ella:

A nivel mundial la trucha posee una alta demanda para su consumo debido a su accesibilidad en el mercado, la realidad en el Ecuador, es que su consumo es relativamente bajo, esto se debe a su limitada forma de preparación, pues en la actualidad se conoce dos métodos representativos de cocción para este pez, el frito y a la plancha, en muy pocas ocasiones se presenta en elaboraciones con cocciones en salsas.

Ahora bien, tal y como se había señalado en la introducción, es necesario hacer una correlación de la teoría con el campo de acción, logrando de esta manera un análisis de los resultados obtenidos durante el proceso de la investigación. Para ello, se usó tres técnicas metodológicas específicas para la recolección de información, entre ellas: la observación, la entrevista y la encuesta. Con los datos obtenidos con estos métodos se pretende relacionar la propuesta de investigación con el problema enfrentado.

Se determinó que los problemas existentes en la empresa se derivan por diversos factores, ya sean estas responsabilidades directas de la entidad o del personal operativo. Logrando evidenciar directamente las dificultades del área de cocina, tal como se evidencia a continuación:

Teniendo en cuenta a Bunge M. (727), "en la medida en que tratamos de describir y de explicar aquello que estamos observando. Al final de una observación científica nos dotamos de algún tipo de explicación acerca de lo que hemos captado, al colocarlo en relación con otros datos y con otros conocimientos previos". Se pudo observar la mala organización del espacio gastronómico como se muestra en el grafico 1, es lo que se debe cumplir en un $100 \%$ en comparación a lo percibido en el restaurante, presentado en el grafico 2, generando un declive en los procesos empleados. 
Gráfico 1» Procesos satisfactorios

\begin{tabular}{ccc}
\hline \hline \multicolumn{3}{c}{ PORCENTAJE IDÓNEO PARA UN PROCESO } \\
ACEPTABLE
\end{tabular}

Fuente: Gómez (1999).

Gráfico 2 » Diagnóstico de procesos aplicados

\begin{tabular}{ccc}
\hline \hline \multicolumn{3}{c}{ PORCENTAJE OBTENIDO EN EL PROCESO } \\
APLICADO \\
\hline $\begin{array}{c}\text { Elementos } \\
\text { del producto }\end{array}$ & $\begin{array}{c}\text { Sistema de } \\
\text { restauración }\end{array}$ & Estandarización \\
\hline $100 \%$ & $70 \%$ & $5 \%$ \\
\hline \hline
\end{tabular}

PORCENTAJE OBTENIDO DEL PROCESO QUE SE APLICA

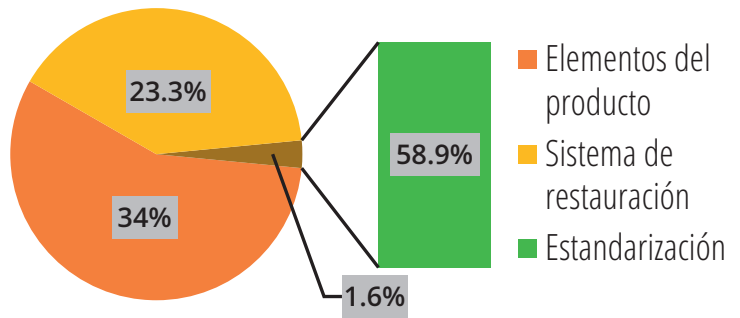

Se puede evidenciar la comparación de ambos gráficos presentados anteriormente la necesidad de implementar los procesos y estandarizaciones fundamentales, que gestionados correctamente crean un valor al cliente y pueden garantizar excelentes resultados en el restaurante corriendo una $41.1 \%$ la falta de aplicación de estos procesos como se detalla en el siguiente gráfico.

Se conoce como estandarización al proceso mediante el cual se realiza una actividad de manera standard o previamente establecida, por consiguiente, en la entrevista efectuada a la administradora del restaurante se pudo determinar la no utilización de hojas de rutas y fichas de costos, base imprescindible para la ejecución de la estandarización. A continuación, en el gráfico 3 permite verificar el antes y después de implementar la estandarización, información recogida desde el diagnóstico inicial en cotejo con la implementación realizada, se manifiesta la diferencia existente entre ambos puntos.

Gráfico 3 » Estandarización previa y posterior

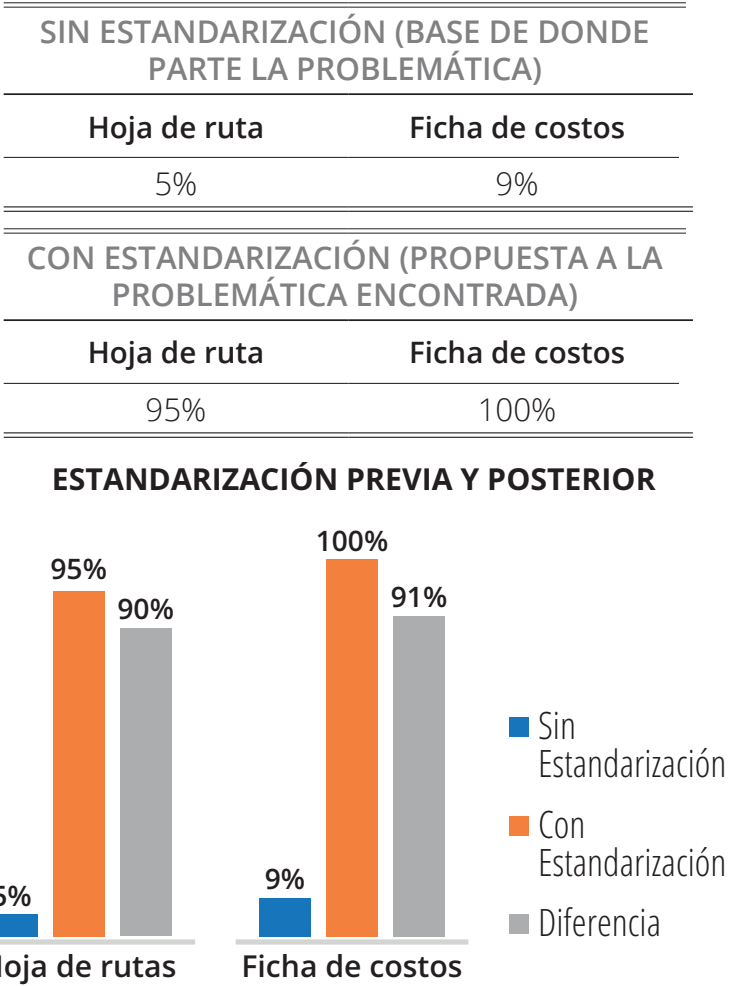

Se aplicó una encuesta censal, realizada al personal operativo del área de cocina del restaurante de la Hacienda/Hostería Dos Chorreras, dando como resultado que las nueve personas que laboran en esta área tienen noción necesaria sobre la ficha estándar. El cual no emplean estos conocimientos en los procesos de elaboración, a pesar que saben usar una ficha estándar correctamente. Por lo que se consideró necesario implementar este método técnico para así mejorar la producción al momento de recrear como se indica en el grafico 4, llevando al comensal un plato de calidad siempre.

Sin embargo, esta técnica no había sido establecida lo cual afecta al proceso de producción. Por lo cual se debe tener en cuenta que la estandarización es importante en todo lugar de producción de alimentos y bebidas. 


\section{Gráfico 4 » Implementación de la ficha estándar al personal operativo}

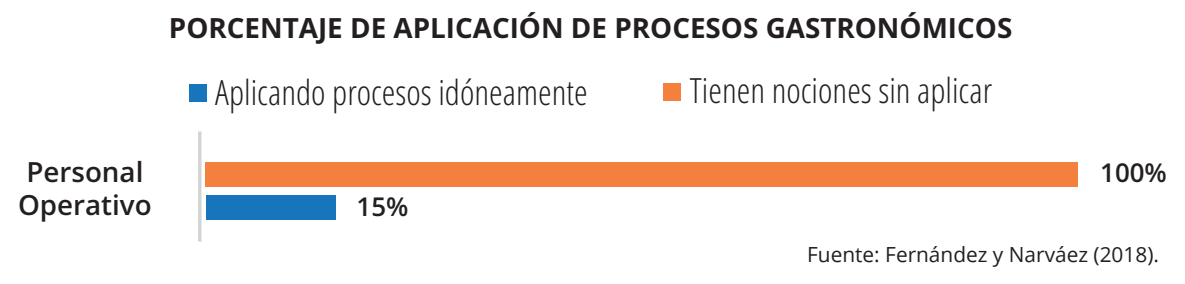

Para el proceso de estandarización se usó diferentes métodos técnicos como son: base de datos, fichas estándar y bitácoras que ayudan a desarrollar el tema de estandarización el cual posteriormente de documentar, entregadas a la administradora, así como al personal operativo de la sección "Truchas y Mariscos" del área de cocina del restaurante de la Hacienda/Hostería Dos Chorreras. Se debe tener en cuenta que la fijación del costo es punto relevante teniendo en cuenta los costos fijos, variables y operativos para luego analizar y obtener el precio de venta al público relacionado cubriendo todos los gastos y generar ganancias.

\section{Propuesta de investigación}

"Antiguamente se realizaban platos con mucha comida, con una pesada salsa y una decoración recargada. Todo esto ha quedado desfasado y se ha sustituido por una presentación artística, con diversidad y color, donde se reconoce a la perfección cada ingrediente en el plato" Fernández (2017).

La gastronomía está plasmada de acuerdo al conocimiento gastronómico del cocinero, así como las ganas de presentar diferentes productos al mundo, teniendo como resultado la cocina actual. Al momento de realizar el diseño de los seis platos fuertes de autor se tomó en cuenta la aplicación de diferentes técnicas, formas y cortes de los géneros, alternando ingredientes, combinando sabores y dando diferentes formas de presentación y decoración. En el diseño de los platos fuertes de autor se tiene como objetivo que al comensal se cautive por el plato a primera vista debido a el esquema de colores usados y su aroma pertinente, así como, al degustar sienta en su paladar distintos sabores y texturas.

Se plantea la técnica de estandarización ya que es una forma de demostrar que es funcional e idónea al momento de implementar un plato en el menú. Teniendo en cuenta que esta sección es la más rotativa, se puede decir que, de cada diez comensales, siete se sirven los platillos de esta sección. El diseño de los seis platos fuertes de autor estandarizados tiene como finalidad incrementar con una opción más variada en la sección "Truchas y Mariscos" del menú del restaurante de la Hacienda/ Hostería Dos Chorreras.

\section{CONCLUSIONES}

- Una vez concluido el estudio se detectó que la falta de una estandarización no solo afecta al personal operativo, sino también al área de administración porque no tienen el costo real de sus platos.

- Las encuestas realizadas al personal operativo sobre la aceptación del uso de la ficha estándar dieron resultados positivos indicando el $100 \%$ de aceptación.

- El presente estudio resalta la importancia de una estandarización para una empresa de producción alimentos.

- La planificación de la socialización ayudó para un mejor entendimiento y aprendizaje al personal operativo de cocina, bodeguero y propietarios sobre la estandarización y propuesta de seis platos de autor.

- La degustación de los dos platos fuertes de autor dio excelentes resultados pues se logró el compromiso tanto con los propietarios de la empresa y el personal operativo.

- El resultado positivo de la investigación se traduce en términos de viabilidad del proyecto de investigación. 


\section{RECOMENDACIONES}

- Con la implementación de la estandarización del menú de la sección" Truchas y Mariscos" se sugiere continuar fomentando entre el personal operativo de cocina para conseguir resultados homogéneos y de posible replica.

- Se recomienda diseñar diferentes opciones de las guarniciones de los platos ya que son repetitivas entre ellas con respecto a los platos del menú.

- Entregar dos uniformes completos para los empleados. Con la finalidad de que exista rotación y limpieza.

- Promover un mayor control de limpieza general del área de cocina. Ya que no existe un cronograma de actividades para la limpieza de la infraestructura de la cocina.

- Comprometer al personal manipulador con la importancia de usar la ficha estándar de forma continua.

- Fortalecer y mejorar en los componentes en los que se encontró deficiencias como en la iluminación, ventilación.

- Control en el almacenamiento ya que resulta perjudicial para la empresa dando de baja alimentos semanalmente por el mal manejo de la materia prima.

\section{BIBLIOGRAFÍA}

Baculima X. \& Quizhpi D. (2017). PROPUESTA DE APLICACIÓN DE TÉCNICAS DE CORTES Y MÉTODOS DE COCCIÓN EN TRES TIPOS DE PESCADOS DE RIO EN RECETAS DE COCINA DE AUTOR. Recuperado de: http://dspace. ucuenca.edu.ec/bitstream/123456789/28893/1/ Monograf\%C3\%ADa.pdf

Bunge Mario (1969), La investigación científica: su estrategia y su filosofía. Versión castellana de Manuel Sacristan. Ediciones Ariel, Colección "Convivium", Barcelona Pg 727

Secretaria de Economía, S. (2015). ¿Qué es la Estandarización?. México: Secretaría de Economía del Gobierno de México. Recuperado de: https:// www.gob.mx/se/articulos/que-es-la-estandarizacion

Fernández, D.. (2017). Cocina creativa o de autor. Madrid, España: CEP, S.L.

Gómez, R. Tendencias actuales en restaurantes. EAEHT. Ciudad de la Habana. Cuba. 1999.

Hernández S. (2010). Diseño mixto de investigación. Compilación. Blogspot en línea. Recuperado de: $\quad$ http://investigacionmixtablog.blogspot. com/2016/03/podemos-definir- el-enfoque-mixto-comoun.html

Rodríguez, E. (2018). 7 secretos que los restaurantes de éxito no quieren que sepas. Alicante, España: Sitio web, Eloy Rodríguez.com. Recuperado de: https:// eloyrodriguez.com/ficha-tecnica-receta-plantilla/ 